Article

\title{
Serum 25-Hydroxyvitamin D Concentrations and Indicators of Mental Health: An Analysis of the Canadian Health Measures Survey
}

\author{
Filmer Chu ${ }^{1, *(\mathbb{D})}$, Arto Ohinmaa ${ }^{2}{ }^{\mathbb{D}}$, Scott Klarenbach ${ }^{1}$, Zing-Wae Wong ${ }^{1}$ and Paul Veugelers ${ }^{2}$ \\ 1 Department of Medicine, Faculty of Medicine and Dentistry, University of Alberta, \\ Edmonton, AB T6G 2G3 Canada; swk@ualberta.ca (S.K.); zingwae@ualberta.ca (Z.W.) \\ 2 School of Public Health, University of Alberta, Edmonton, AB T6G 1C9, Canada; \\ aohinmaa@ualberta.ca (A.O.); paul.veugelers@ualberta.ca (P.V.) \\ * Correspondence: Filmer@ualberta.ca; Tel.: +1-780-495-2244
}

Received: 25 August 2017; Accepted: 9 October 2017; Published: 13 October 2017

\begin{abstract}
The main function of vitamin D is calcium homeostasis. However, emerging evidence has correlated adequate serum 25-hydroxyvitamin $\mathrm{D}(25(\mathrm{OH}) \mathrm{D})$ concentrations with better mental health. The objective of this study is to investigate the association of serum $25(\mathrm{OH}) \mathrm{D}$ concentrations with indicators of mental health such as depression, anxiety, and stress. Associations of serum 25(OH)D concentrations with four indicators of mental health were examined using ordered logistic regression models with increasing specificity that account for demographics, socio-economic status, and health. Margin effects are used to determine the probability of the average adult Canadian being in the best mental health state by groupings of serum $25(\mathrm{OH}) \mathrm{D}$ concentrations. A robust association between serum $25(\mathrm{OH}) \mathrm{D}$ concentrations and the indicators of mental health were observed. In the fully adjusted ordered logistic model, an average Canadian appeared more likely to experience better mental health when serum 25(OH)D concentrations were higher. This study adds to the weight of the existence of an association between vitamin D status and mental health, but, as this study is cross sectional, it does not establish causality. Due to the low risk of harm from toxicity and the relative modest costs of vitamin D supplements, more research to establish the effectiveness and causality of this relationship is recommended.
\end{abstract}

Keywords: vitamin D; Canada; depression; mental health

\section{Introduction}

Mental health may be impacted by a range of mood disorders that effect both thinking and behavior such as depression, anxiety, and schizophrenia, as well as in substance use disorder. One in every five Canadians experiences a mental health problem, and by the time Canadians reach 40 years of age, one in every two Canadians have or have had a mental illness [1]. Reducing this disease burden will have a cascading effect on the health care system, from reduce direct cost on mental health professionals to the indirect cost of reduced substance abuse [2]. This study focuses on three indicators of mental health: depression, anxiety, and stress. The notion that relative inexpensive vitamin D supplements can improve mental health outcomes warrants an exploratory Canadian study. Despite vitamin D fortification, Canadians are not getting enough vitamin $\mathrm{D}$ to benefit from the potential protective effects especially during the winter [3].

The main function of vitamin $\mathrm{D}$ is to regulate the absorption (homeostasis) of calcium for better bone health $[4,5]$. However, emerging evidence has correlated adequate levels of serum 25-hydroxyvitamin D (25(OH)D) concentrations with better scores on indicators of mental health [6]. Active vitamin D binds to vitamin D receptors (VDR) to regulate physiologic functions of the body 
such as emotional well-being and stress. VDRs are found in more than 30 cell types throughout the body [7], including neuronal and glial cells [8] in the cortex and hippocampus, which have been implicated in the pathophysiology of mood [9]. Experiments on rodents have demonstrated alterations to brain function and behavior due to vitamin D deficiency [10]. Active vitamin D also regulates tyrosine hydroxylase, which in turn regulates the production of the mood regulating neurotransmitters, norepinephrine and dopamine [11]. The absolute or relative lack of norepinephrine is associated with most, if not all, types of mental health outcomes [11]. Lower dopamine levels are associated with mental health issues such as diminished motivation and psychomotor retardation [12]. Vitamin D may indirectly regulate mood by stimulating genes that produce neurotransmitters that relieve depressive emotions [13]. This biological link between vitamin D and mood raises the hypothesis of whether adequate vitamin $\mathrm{D}$ levels are associated with reduced probability of mental health illness.

Previous studies have found evidence that low serum 25(OH)D concentrations are associated with depression [14]. This includes studies from various countries showing a positive relationship between serum 25(OH)D concentrations and mental health indicators $[15,16]$ and two community based samples of older Canadians [16,17]. It has been suggested that over one third of the Canadian population have suboptimal serum $25(\mathrm{OH}) \mathrm{D}$ concentrations [15]. If a causal relationship exists, this may be of considerable importance given the high rate of mental health illness in Canada [1]. This study investigates the relationship between serum $25(\mathrm{OH}) \mathrm{D}$ concentrations and mental health indicators using an established survey among a large sample that is representative of Canadians.

\section{Methodology and Dataset}

The Canadian Health Measures Survey (CHMS) is a cross-sectional survey conducted every two years, developed and conducted by Statistics Canada in partnership with Health Canada and the Public Health Agency of Canada. Currently, three cycles are available from year 2007 to 2013. The CHMS covers the population aged 3 to 79 years living in the ten provinces. The data excludes people who are living in the three territories, living on reserves and other Aboriginal settlements, full-time members of the Canadian Forces, part of the institutionalized population, and residents of certain remote regions. Altogether, these exclusions represent approximately $4 \%$ of the Canadian population [18]. This study will include Canadians over the age of 18 and non-pregnant (sample size 7518). Sixteen mobile examination centers across Canada with trained professional collected blood samples for the assessment of serum $25(\mathrm{OH}) \mathrm{D}$ concentrations (expressed in $\mathrm{nmol} / \mathrm{L}$ ) constituting the exposure of interest.

The gold standard assessment of mental health and well-being is an assessment from a mental health professional. However, this is unfeasible, impractical, and costly to obtain for a nationally representative dataset. This study uses four proxy measures of depression, anxiety, and stress. The first proxy exists in two cycles of the CHMS contains questions designed to derive the various components of the Health Utility Index 3 (HUI3). The HUI3 was developed in Canada and was designed to quantify overall health using eight attributes measured on a scale between one and six, where one indicated a better health state. The emotional attribute is extracted as one of proxies of depression/anxiety and was determined by the following question "Would you describe yourself as being usually: happy and interested in life; somewhat happy; somewhat unhappy; unhappy with little interest in life; so unhappy that life is not worthwhile". The remaining three proxies are available in all three cycles of the CHMS. The second proxy is self-perceived mental health which, was measured by the question "In general, would you say your mental health is: Excellent; very good; good; fair; poor". The remaining two proxies are self-perceived stress and self-perceived general health. Stress is measured by asking the respondent "Thinking about the amount of stress in your life, would you say that most days are: not at all stressful; not very stressful; a bit stressful; quite a bit stressful; extremely stressful". The final proxy is a measure of general health but it can be argued that the belief of one's general health is associated with their mental health and as such this measure will be used as a tertiary proxy of depression, anxiety, and stress. General health is measured by asking the respondent "in general, would you say your 
health is: Excellent; very good; good; fair; poor". All four proxies are modelled with the initial two cycles stacked and resampled with the bootstrap weights provided by Statistics Canada to increase the statistical power and to provide a representative sample of the Canada Adult population. Proxies with three cycles are independently restacked and resampled with the appropriate bootstrap weights as a robustness check.

The relationship between vitamin D status and indicators mental health is entangled by many observable confounders including demographics, socio-economic status, chronic conditions, smoking and drinking, illicit drug use and labor force status. Demographic controls in this study include age, sex, marital status, education, and ethnicity. Socio-economic status is controlled with household income and if the respondent is a student. Lifestyle controls consist of smoking, drinking, drug use (both prescription and street drugs). Health conditions are controlled with binary indicators for chronic and acute conditions (based on self-reported official diagnostics). The specific variables are also described in Table 1. Ordered logistic regression analyses will be performed with increasing specificity to ensure robustness of the results. The dependent variables are the mental health proxies as described (Table 2). Each proxy will be used independently with increasing model specifications to ensure robustness of results.

Table 1. Bootstrapped weighted descriptive statistics of the Canadian adult population.

\begin{tabular}{cc}
\hline Control Variables & Measure \\
\hline average age (years) & 45 \\
average household income (Canadian dollars) & 77,548 \\
average weight $(\mathrm{kg})$ & 78 \\
average height $(\mathrm{cm})$ & 169 \\
males & $50 \%$ \\
marital status: married & $51 \%$ \\
education & - \\
secondary or less & $30 \%$ \\
colleague/trades/certificates & $45 \%$ \\
university or higher & $25 \%$ \\
white racial Origin & $82 \%$ \\
student & $12 \%$ \\
smokes daily & $17 \%$ \\
regular drinker & $68 \%$ \\
used prescription drugs for recreational purposes & $3 \%$ \\
used or tried street drugs & $15 \%$ \\
weight status (as defined by the World Health Organization) & - \\
underweight & $2 \%$ \\
normal weight & $42 \%$ \\
overweight & $35 \%$ \\
obese & $20 \%$ \\
\hline
\end{tabular}

Unobservable confounders such as Seasonal Affect Disorder (SAD) are periodic major depression occurs in some people during late fall to early spring, which may affect the indicators of mental health. Some patients have the opposite occurrence: depressive symptoms during spring and summer. Regardless of the season, the depression episodes occur during the same seasons every year [19]. The proposed datasets accounts for seasonality effects by uniformly distributing the 16 mobile examination center collection sites by region between the collection years [18]. 
Table 2. Indicators of mental health.

\begin{tabular}{cc}
\hline Dependent Variables & \% of Total Population \\
\hline emotional problems (HUI3) & - \\
life not worthwhile & $0.3 \%$ \\
very unhappy & $0.7 \%$ \\
somewhat unhappy & $3.1 \%$ \\
somewhat happy & $17.5 \%$ \\
happy in life & $78.4 \%$ \\
self-perceived mental health & - \\
poor & $0.9 \%$ \\
fair & $4.3 \%$ \\
good & $21.9 \%$ \\
very good & $38.8 \%$ \\
excellent & $34.1 \%$ \\
self-perceived stress & - \\
extremely & $3.2 \%$ \\
quite a bit & $16.8 \%$ \\
a bit & $44.0 \%$ \\
not very & $26.4 \%$ \\
not at all & $9.7 \%$ \\
self-perceived health & - \\
poor & $2.5 \%$ \\
fair & $8.8 \%$ \\
good & $36.4 \%$ \\
very good & $38.4 \%$ \\
excellent & $13.8 \%$ \\
\hline & \\
\hline &
\end{tabular}

\section{Results}

The characteristics of the population (Table 1) indicate an average age for adult Canadians (Canadians over the age of 18, excludes all pregnant females) is 45 with an average household income of $\$ 77,550$. The average weight and height are $78 \mathrm{~kg}$ and $169 \mathrm{~cm}$, respectively. Approximately $50 \%$ of Canadians are married. There is an equal split between males and females. Approximately $25 \%$ of the population graduated with an university degree or higher. Approximately $17 \%$ of the population smokes daily, and $68 \%$ consume alcoholic beverages on a regular basis. Over $50 \%$ of the population is considered inactive. Approximately $42 \%$ of Canadians have a normal weight. The indicators of mental health (Table 2) indicate that approximately $78 \%$ of Canadians are happy in life, and $34 \%$ have excellent self-perceived mental health. The indicator of self-perceived stress seems quite normally distributed, with $44 \%$ of Canadians having "a bit" of self-perceived stress. Most Canadians have a self-perceived health of being in the good, very good, and excellent category.

Emotional health has a positive association with serum $25(\mathrm{OH}) \mathrm{D}$ concentrations across all models (Table 3). For every $25 \mathrm{nmol} / \mathrm{L}$ increase of serum 25(OH)D concentrations, there is an average of a 1.16-unit increase in log odds (or a $76 \%$ increase of being in the best emotional health category of the HUI3 index, calculated by converting log odds to probability) across all models that controls for demographics, socioeconomic status, lifestyle choices, and health of the respondent. Similar increase in predictive probability of being in a better mental health state are found for self-perceived mental health (75\%), self-perceived stress (75\%), and self-perceived general health $(76 \%)$ for every $25 \mathrm{nmol} / \mathrm{L}$ increase of serum $25(\mathrm{OH}) \mathrm{D}$ concentrations in the blood stream. 
Table 3. Coefficients for the association of serum $25(\mathrm{OH}) \mathrm{D}$ concentrations (per $25 \mathrm{nmol} / \mathrm{L}$ increase) with mental health indicators in various regression models.

\begin{tabular}{cccccc}
\hline Dependent Variable & Unadjusted Model & +Demographics & +Socioeconomic & +Life Style & +Health \\
\hline Emotional health & $1.24^{* * *}(0.06)$ & $1.19^{* * *}(0.06)$ & $1.17^{* * *}(0.06)$ & $1.15^{* * *}(0.05)$ & $1.16^{* * *}(0.06)$ \\
Mental Health & $1.10^{* * *}(0.04)$ & $1.10^{* *}(0.04)$ & $1.09^{* *}(0.04)$ & $1.07^{*}(0.04)$ & $1.08^{*}(0.04)$ \\
Stress & $1.09^{* *}(0.05)$ & $1.10^{* *}(0.04)$ & $1.10^{* *}(0.05)$ & $1.09^{*}(0.05)$ & $1.10^{* *}(0.05)$ \\
General Health & $1.23^{* * *}(0.04)$ & $1.20^{* * *}(0.04)$ & $1.19^{* * *}(0.04)$ & $1.16^{* * *}(0.04)$ & $1.17^{* * *}(0.04)$ \\
\hline
\end{tabular}

$*, * *, * * *$ indicates $1 \%, 5 \%, 10 \%$, significance levels, respectively. Bootstrap standard errors in parentheses.

The adjusted probability of being in the best health state for both the HUI3 emotional health category and self-perceived stress category increases with higher serum 25(OH)D concentrations. This probability is based on the average Canadian as defined previously. Similar adjusted probabilities of being in the best health state were found for self-perceived mental health and self-perceived general health. The probability of the average Canadian being in the best mental health state has an upward tendency as serum $25(\mathrm{OH}) \mathrm{D}$ concentrations increases and more so when moving from very low levels to $100 \mathrm{nmol} / \mathrm{L}$. This is also when the confidence interval is narrowest suggesting a higher confidence in the prediction (Figure 1).
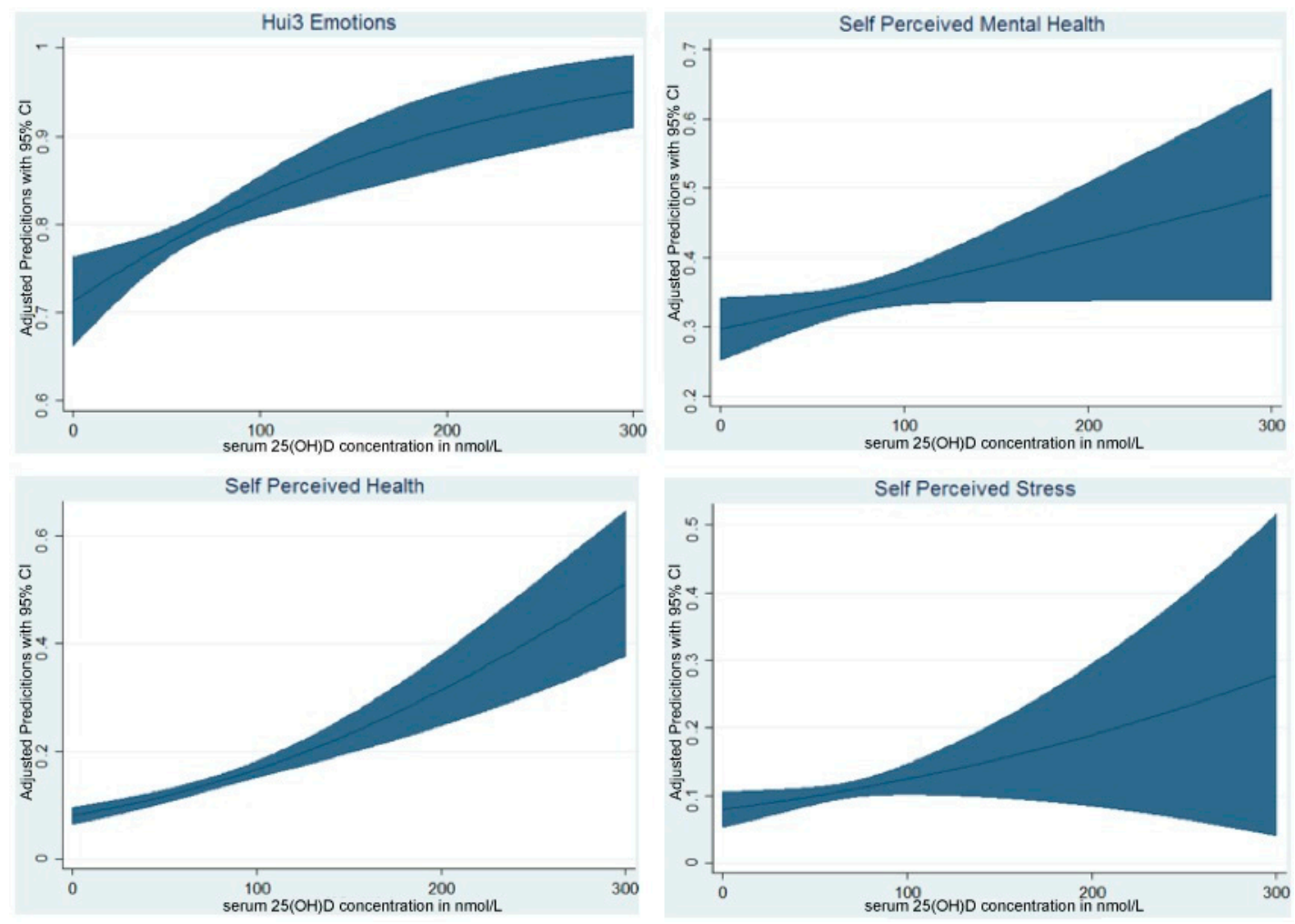

Figure 1. Adjusted probability with $95 \%$ confidence interval of being in the best mental health state by serum $25(\mathrm{OH}) \mathrm{D}$ concentrations.

\section{Robustness}

All outcome variables of interest (self-perceived mental health, general health, and stress) available in Cycle 3 underwent the same regression models with all three cycles stacked with the appropriate Statistics Canada bootstrap weights. All results are consistent if not better compared with the main analysis. The confidence intervals are narrower when the three cycles are stacked. This is most likely due to the increase sample size that resulted in an increase of statistical power. All robustness results are available upon request from the corresponding author. 


\section{Limitations}

There are two important limitations to this study. The first is that this study can only determine an association and not a causal effect. In other words, it is unclear whether vitamin D produces better scores on the indicators of mental health or if lower depression and anxiety leads to higher serum 25(OH)D concentrations in the blood from better nutrition and/or outdoor activities. In other words, someone with depression and anxiety may not venture outside (less exposure to sunlight), and someone without depression and anxiety might be more active and go outside more. The authors attempted to estimate the casual affect through instrumental variable regression methods but were unable to find a valid instrument in the dataset, with the inability to control for all unobservable confounders such as nutrient-nutrient interactions and other individual heteroscedasticities.

The second major limitation is the method of ascertaining mental health. To accurately measure mental health states, a standard method completed by health professionals would be required. However, this is unrealistic, impractical, and costly for a nationally representative dataset. It is also very likely that due to the self-perceived nature of the questionnaires, these proxies are likely to be underestimated. Some of the proxies utilized in this study stem from health-related quality of life measurements, and we acknowledge that these may not be as good compared to mental health related quality of life instruments. Linking of other datasets has also been considered, but due to privacy rules with the CHMS dataset, this was not an option.

\section{Discussion}

This study reveals robust positive associations between serum 25(OH)D concentrations and indicators of mental health (depression and anxiety) after controlling for demographics, socio-economic status, chronic conditions, smoking and drinking, illicit drug use, and labor force status. This study also found that higher serum $25(\mathrm{OH}) \mathrm{D}$ concentrations by each increment of $25 \mathrm{nmol} / \mathrm{L}$ increases the probability of the average Canadian to be in the best mental health and general health state by an average of $76 \%$.

Accurate measures of mental health and well-being ideally would include an assessment from a mental health professional which is unfeasible, impractical, and costly to obtain for a large nationally representative study sample. This study uses four established proxy measures of mental health. However, there are inherent biases, such as response bias due to the nature of the question. These biases must be weighed against the possible benefits in order to assess the potential overall benefit.

Vitamin D is generally well tolerated, and adverse events and toxicity are rare when it is taken appropriately. To raise serum $25(\mathrm{OH}) \mathrm{D}$ concentrations to optimal amounts as shown in previous studies, Canadians would need a daily intake of 1000-4000 IU/day. However, Canadians on average can only obtain 200-300 IU / day from food sources [20] and live at high latitudes and thus have less sun exposure and subcutaneous vitamin D synthesis. Canadians would need to obtain the daily intake of vitamin $\mathrm{D}$ in arrays through supplements over a long period of time to see any benefits due to the short half-life of vitamin D (3 weeks).

Studies in countries such as China, England, Europe, Japan, The Netherlands, and United States have shown a robust relationship between vitamin D status and depression $[6,15,16,21,22]$. Two studies in a sample of older Canadians participating in a preventive health program showed that higher serum $25(\mathrm{OH}) \mathrm{D}$ concentrations were associated with improved health related quality of life [16,17]. Those observations, however, cannot be generalizable because they were in a selected group. The present study is the first to extend the finding to the entire Canadian adult population. Given the burden of mental health issues in Canada, and the large proportion of the Canadian population with suboptimal serum $25(\mathrm{OH}) \mathrm{D}$ concentrations [15], the present study adds to the support of the notion that relative inexpensive vitamin D supplements can prevent mental health problem and improve mental health outcomes is compelling and warrants a definitive study to determine effectiveness. 


\section{Reference}

Acknowledgments: The authors would like to thank Sentil Senthilselvan (University of Alberta. School of Public Health) and Irene Wong (Statistics Canada RDC Edmonton) for their help in understanding the complexity of the CHMS dataset. They would also like to thank Haifang Huang (University of Alberta, Department of Economics), Philip Jacobs (University of Alberta, Department of medicine), and Alain Lesage (University of Montreal, Department of Psychiatry) for their constructive criticism during the Candidacy Exam. S.K. is supported by the Kidney Health Research Chair and the Division of Nephrology at the University of Alberta. P.J.V. holds a Canada Research Chair in Population Health, an Alberta Research Chair in Nutrition and Disease Prevention, and an Alberta Innovates Health Scholarship.

Author Contributions: The corresponding author led the study, but all other authors equally helped to conceive and design the study and develop the manuscript.

Conflicts of Interest: The authors declare no conflict of interest. The research was supported by funds to Canadian Research Data Centre Network (CRDCN) from the Social Sciences and Humanities Research Council (SSHRC), the Canadian Institute for Health Research (CIHR), the Canadian Foundation for Innovation (CFI), and Statistics Canada. Although the research and analysis are based on data from Statistics Canada, the opinions expressed do not represent the views of Statistics Canada.

\section{References}

1. Smetanin, P.; Briante, C.; Stiff, D.; Ahmad, S.; Khan, M. Life and Economic Impact of Major Mental Illnesses in Canada, 2011 to 2041; Mental Health Commission of Canada: Toronto, ON, Canada, 2011.

2. Buckley, P.F.; Miller, B.J.; Lehrer, D.S.; Castle, D.J. Psychiatric Comorbidities and Schizophrenia. Schizophr. Bull. 2009, 35, 383-402. [CrossRef] [PubMed]

3. Calvo, M.S.; Whiting, S.J.; Barton, C.N. Vitamin D fortification in the United States and Canada: Current status and data needs. Am. J. Clin. Nutr. 2004, 80, 1710S-1716S. [PubMed]

4. Institute of Medicine (US) Committee to Review Dietary Reference Intakes for Vitamin D and Calcium. Dietary Reference Intakes for Calcium and Vitamin D; Ross, A.C., Taylor, C.L., Yaktine, A.L., del Vallea, H.B., Eds.; National Academies Press: Washington, DC, USA, 2011.

5. Nussey, S.; Whitehead, S. Endocrinology: An Integrated Approach; BIOS Scientific Publishers Limited: London, UK, 2001.

6. Wilkins, C.; Sheline, Y.; Roe, C.; Birge, S.; Morris, J. Vitamin D deficiency is associated with low mood and worse cognitive performance in older adults. Am. J. Geriatr. Psychiatry 2006, 14, 1032-1040. [CrossRef] [PubMed]

7. Holick, M. Vitamin D deficiency. N. Engl. J. Med. 2007, 357, 266-281. [CrossRef] [PubMed]

8. Eyles, D.; Smith, S.; Kinobe, M.; Hewison, M.; McGrath, J. Distribution of the vitamin D receptor and 1 alphahydroxylase in human brain. J. Chem. Neuroanat. 2005, 29, 21-30. [CrossRef] [PubMed]

9. Fernandes de Abreu, D.; Eyles, D.; Feron, F. Vitamin D, A neuro-immunomodulator: Implications for neurodegenerative and autoimmune diseases. Psychoneuroendocrinology 2009, 34, S265-S277. [CrossRef] [PubMed]

10. Groves, N.J.; Kesby, J.P.; Eyles, D.W.; McGrath, J.J.; Mackay-Sim, A.; Burne, T.H.J. Adult vitamin D deficiency leads to behavioural and brain neurochemical alterations in C57BL/6J and BALB/c mice. Behav. Brain Res. 2013, 241, 120-131. [CrossRef] [PubMed]

11. Schildkraut, J. The catecholamine hypothesis of affective disorders: A review of supporting evidence. Am. J. Psychiatry 1965, 122, 509-522. [CrossRef] [PubMed]

12. Dailly, E.; Chenu, F.; Renard, C.E.; Bourin, M. Dopamine, depression and antidepressants. Fundam. Clin. Pharmacol. 2004, 18, 601-607. [CrossRef] [PubMed]

13. Eyles, D.; Almeras, L.; Benech, P.; Patatian, A.; Mackay-Sim, A.; Mcgrath, J.; Feron, F. Developmental vitamin $D$ deficiency alters the expression of genes encoding mitochondrial, cytoskeletal and synaptic proteins in the adult rat brain. J. Steroid Biochem. Mol. Biol. 2007, 103, 538-545. [CrossRef] [PubMed]

14. Polak, M.A.; Houghton, L.A.; Reeder, A.I.; Harper, M.J.; Conner, T.S. Serum 25-Hydroxyvitamin D Concentrations and Depressive Symptoms among Young Adult Men and Women. Nutrients 2014, 6, 4720-4730. [CrossRef] [PubMed]

15. Anglin, R.E.S.; Samaan, Z.; Walter, S.D.; McDonald, S.D. Vitamin D deficiency and depression in adults: Systematic review and meta-analysis. Br. J. Psychiatry 2013, 202, 100-107. [CrossRef] [PubMed] 
16. Chao, Y.S.; Ekwaru, J.P.; Ohinmaa, A.; Griener, G.; Veugelers, P.J. Vitamin D and health-related quality of life in a community sample of older Canadians. Qual. Life Res. 2014, 23, 2569-2575. [CrossRef] [PubMed]

17. Ekwaru, J.P.; Ohinmaa, A.; Veugelers, P. The effectiveness of a preventive health program and vitamin D. Qual. Life Res. 2016, 25, 661-668. [CrossRef] [PubMed]

18. Statistics Canada. Canadian Health Measures Survey (CHMS) Data User Guide: Cycle 1. [Internet]. 2011. Available online: http://www23.statcan.gc.ca/imdb-bmdi/document/5071_D2_T1_V1-eng.htm (access on 15 July 2016).

19. Staff, M.C. Seasonal Affective Disorder. [Internet]. 2014. Available online: http://www.mayoclinic.org/ diseases-conditions/seasonal-affective-disorder/basics/definition/con-20021047 (accessed on 20 July 2016).

20. Grant, W.B.; Whitinh, S.J.; Schwalfenberg, G.K.; Genuis, S.J.; Kimball, S.M. Estimated economic benefit of increasing 25-hydroxyvitamin D concentrations of canadians to or above $100 \mathrm{nmol} / \mathrm{L}$. Derm. Endocrinol. 2016, 8, e1248324. [CrossRef] [PubMed]

21. Okere, O.; Singh, A. The roleofvitaminDinthepreventionoflate-lifedepression. J. Affect. Disord. 2016, 198, $1-14$.

22. Lapid, M.; Cha, S.; Takahashi, P. Vitamin D and depression in geriatric primary carepatients. Clin. Interv. Aging 2013, 8, 509-514. [CrossRef] [PubMed]

(C) 2017 by the authors. Licensee MDPI, Basel, Switzerland. This article is an open access article distributed under the terms and conditions of the Creative Commons Attribution (CC BY) license (http://creativecommons.org/licenses/by/4.0/). 